\title{
THE EFFECT OF NIGELLA SATIVA LINN (KALAJIRA) EXTRACT ON GENTAMICIN- INDUCED NEPHROTOXICITY IN EXPERIMENTAL RATS
}

\author{
QUADIR R ${ }^{1}$, KHAN MI $^{2}$, EVA EO $^{3}$, RAHMAN H ${ }^{4}$, AHASAN F 5 , HASAN MJ 6
}

\begin{abstract}
The pathogenesis of gentamicin-induced nephrotoxicity has shown to generate oxygen free radicals. Several free radical scavengers are well recognized to ameliorate the nephrotoxicity. The seeds and oil of nigella sativa were reported to possess strong antioxidant properties and was effective against disease and chemically-induced hepatotoxicity and nephrotoxicity. The experiments were carried out in two parts, Experiment I and Experiment II, on a total of 35 rats of 8-12 weeks old and weighing between 200 and 230g. Nephrotoxicity and amelioration of nephrotoxicity was evaluated by measurement of concentrations of serum creatinine. The rats were induced nephrotoxicity by subcutaneous injection of gentamicin $100 \mathrm{mg} / \mathrm{kg} /$ day for 9 days and were sacrificed on $10^{\text {th }}$ day. The results indicated that gentamicin treatment caused marked renal tubular damage significant increase $(P<0.001)$ of serum creatinine concentrations when compared to those of control. When $n$-hexane extract of $N$.sativa was administered as low and high dose with gentamicin and compared with the gentamicin treated groups, it was found that in these two groups there was significant decrease $(P<0.001)$ of serum creatinine levels. When these two groups were compared with each other, it was observed that more amelioration occurred significantly in high dose treatment group than in the low dose treatment group.

This study established that oral administration of n-hexane extract of $N$. sativa was able to produce considerable improvement from the nephrotoxic action of gentamicin in rats. The best amelioration was obtained in high dose treatment. Low dose treatment brought out the least amelioration of them all. Future works could better be directed towards obtaining the specific ingredient and the specific mechanism responsible for nephroprotection. We are hopeful that complete amelioration might not be impossible if given in proper doses or more effectively if we could extract the actual ingredients responsible for nephroprotection and can use them eventually.
\end{abstract}

Key words: serum creatinine, gentamicin, Nigella sativa (Kalajira) seeds.

J Dhaka Med Coll. 2016; 25(2) : 119-123

\section{Introduction:}

Kidney diseases are one of the major health hazards both in terms of morbidity and mortality. Among the kidney diseases, acute renal failure (ARF) in children is a common and serious renal problem in our country ( Salih B J et al.,2009). Approximately 10,000-15,000 patients with ARF die each year in Bangladesh. Among the causes of ARF, the most important one is injury to the renal tubular cells (acute tubular necrosis) by toxin or ischemia.
The kidneys are the essential excretory organs of our body, and regulate the volume of body fluids and its composition (although the intestines, the lungs and the skin take part to some extent) (Ranju G et al., 2011). The internal environment of the body is thus properly maintained. The maintenance of haemodynamics requires the excretion of various harmful product of metabolism. The kidneys bear the greatest responsibility of fluid and electrolyte reabsorption, secretion and

1. Dr. Rukhsana Quadir, Lecturer, Department of Pharmacology, DMC.

2. Prof. Md. Ismail Khan, Professor, Department of Pharmacology, DMC.

3. Dr. Eliza Omar Eva, Associate Professor Department of Pharmacology, DMC.

4. Dr. Hasanur Rahman, Assistant Professor Department of Pharmacology, DMC.

5. Dr. Faizul Ahasan, General Medical Practitioner, The Doctors BD.

6. Dr. Md. Jiaul Hasan, Assistant Professor, Department of Pharmacology, Manikgonj Medical College. Correspondence : Dr. Rukhsana Quadir, Lecturer, Department of Pharmacology, DMC, Mob no. 01714359766 Received: 05 June 2016 
excretion of excess as well as harmful solute and water. Many products of metabolism of daily food intake, drugs and inhaled chemicals get their passage out of the body through the kidneys saving the mankind from many harmful diseases.

The kidneys receive about $20 \%$ of the cardiac output.This high blood supply also makes the kidneys susceptible to toxic injuries by the drugs and toxins. Of the amount of blood going to each kidney, $90 \%$ or more of them is distributed to the cortical areas which contain the renal corpuscles and most parts of the renal tubules. Thus, the exposure to circulating toxic substances is greater for the cortical tubules than that for most of other tissues.

Among the drugs showing nephrotoxicity, the Aminoglycoside gentamicin is well-recognized, reputed to produce renal tubular damage. It is an effective drug because of its effects against gram-negative organisms and has been a favorable choice to the clinicians. Therefore, in spite of the nephrotoxicity it produces, its low cost and reliable activity against all but the most resistant gram-negative aerobes has kept it as one of the most preferable drugs for gramnegative infections (Chambers.,2001).

Several renal insufficiencies have been reported in patients with previously normal blood urea nitrogen (BUN) and urinalysis while on usual or therapeutic doses $(2 \mathrm{mg} / \mathrm{kg} /$ body weight) of gentamicin. Increasing the duration of gentamicin would increase the percentage of patients with nephrotoxic effects. Less than $15 \%$ of the patients receiving Aminoglycosides for 5 days or less developed nephrotoxicity and the percentage was more than $40 \%$ with treatment for 14 days or more.

Some patients treated with gentamicin may progress to ARF, and among them, a significant number had received gentamicin before the onset of the syndrome. 10 to $15 \%$ of all cases of ARF were users of Aminoglycosides.

The traditionally available remedies for gentamicin-induced nephrotoxicity from natural sources from the Southeast Asia may be worth mentioning at this point. Because of local and easy availability of certain herbs, treatment of gentamicin-induced nephrotoxicity with traditional medicine would probably be cheaper and less harmful if effective remedy could be provided.

Nigella sativa Linn. (kalajira) seeds locally called kalajira in Bangladesh is one such herbal product. It is a well-known spice of Southeast Asia, especially in Bangladesh. Its disease alleviating properties have strengthened its use as one of the food ingredients in our country. Its antidiabetic effect, lipid-lowering potentialities (Alam S et al.,2013), and antibacterial potentialities had been observed.

Pharmacologically active constituents in the oil of N. sativa seed were thymoquinine (TQ), dithymoquinine(DTQ) and thymol (TOH) It has been shown that both the fixed oil of $\mathrm{N}$. sativa and thymoquinine, the main compound of the essential oil, inhibit non-enzymetic lipid peroxidation in liposomes and posses strong antioxidant properties. The essential oil of $\mathrm{N}$. sativa Linn. seeds considered to have antioxidant activity shown to modify gentamicin induced nephrotoxicity. Prophylaxis of rats orally with $\mathrm{N}$. sativa extract resulted in a significant decrease in renal microsomal lipid peroxidation, Gama-glulamyl-transpeptidase, hydrogen peroxide and xanthine oxidase. There was significant recovery of renal glutathione content and antioxidant enzymes. There was also reversal in the enhancement of blood urea nitrogen, serum creatinine. N. sativa oil produced a dose dependent amelioration of the biochemical of gentamicin nephrotoxicity. N. sativa increased the amount of reduced glutathione and enhancement of total antioxidant status concentrations in renal cortex. This reduced glutathione no longer cause lipid peroxidation and acts as a free radical scavenger.

With these background information, in this study, attempt has been made to evaluate the nephroprotective role of $\mathrm{N}$. sativa on experimentally induced nephrotoxicity in rats. Gentamicin has been chosen to induce the nephrotoxicity.

\section{Materials \& Method:}

The study was carried out in the Department of Pharmacology, Dhaka Medical College, 
Dhaka on 32 albino rats with Nigella sativa (kalajira) extract. Kalajira were collected from market and its ethanolic extract was prepared in CARS. Gentamycin were collected from Square pharmaceutical. Total 35 albino rats were collected from ICDDR,B Dhaka. They were of either sex, weighing about 150-200 gm. Rats were randomly divided into 5 groups of 7 in each group and the study was carried out in 2 stage, experiment 1 and experiment 2 . In experiment 1 Group A served as control group that received distilled water $1 \mathrm{ml}$ orally and normal rat diet daily for 9 days and Group B received gentamicin subcutaneously $(100 \mathrm{mg} / \mathrm{kg}$ body weight/day) for 9 days. On $10^{\text {th }}$ day blood samples were collected from rats through cardiac puncture, and sent for haematological analysis by automated haematology analyzer. In experiment 2 rats were divided into 3 groups, Group C , Group D and Group E and each group contained 7 rats. Group $\mathrm{C}$ gentamicin subcutaneously $(100 \mathrm{mg} / \mathrm{kg}$ body weight/day) with normal diet for 9 days served as experimental control group. Group D received gentamicin $(100 \mathrm{mg} / \mathrm{kg}$ body weight/day) subcutaneously for 9 days along with N. sativa extract $(10 \mathrm{mg} / \mathrm{kg}$ body weight/day) mixed in 5 $\mathrm{ml}$ deionised water, orally.with normal diet for 9 days and Group E received gentamicin subcutaneously $(100 \mathrm{mg} / \mathrm{kg}$ body weight/day) for 9 days along with $\mathrm{N}$. sativa extract orally (20mg/ kg bodyweight/day) mixed in $5 \mathrm{ml}$ deionised water for 9 days. On $10^{\text {th }}$ day blood samples were collected from rats through cardiac puncture, and sent for haematological analysis by automated haematology analyzer.

\section{Results:}

Obtained data on blood urea and serum creatinine concentration were recorded \& compiled. Data were expressed as mean \pm SEM and tabulated $\&$ presented accordingly in tables $\&$ diagrams. Each diagram represented the Mean \pm SE of a variable of specific group. Each line diagram represented dose dependent change of a variable. Results of intervention groups were compared with that of control group. Statistical analysis of recorded quantitative data was done by ANOVA (F test) \& Student's unpaired t-test. Test result was considered as statistically significant at $\mathrm{P}$ value $<0.05$.

Table-I

Comparison of serum creatinine between Group A and Group B.

\begin{tabular}{lccc}
\hline Group & Number of rats & Mean serum creatinine $(\mathrm{mg} / \mathrm{d})$ & P value \\
\hline A & 7 & $1.72 \pm 0.04$ & $<0.001$ \\
B & 7 & $4.98 \pm 0.38$ & \\
\hline
\end{tabular}

Data were expressed as Mean \pm SE. Statistical analysis were done by One-way ANOVA test and Bonferroni test was performed to compare between groups. Experiment-1Group A (Control) Group $\mathrm{B}$ (Experiment) $\mathrm{n}=$ Total number of subjects $=$ Significant at $\mathrm{P}<0.05$; Not significant $(\mathrm{P}>0.05)$.

Table-II

Comparison of serum creatinine of Group C with Group D and Group E.

\begin{tabular}{lccc}
\hline Group & Number of rats & Mean serum creatinine $(\mathrm{mg} / \mathrm{dl})$ & P value \\
\hline C & 7 & $4.73 \pm 0.32$ & $<0.01$ \\
D & 7 & $3.59 \pm 0.15$ & \\
\hline Group & Number of rats & Mean serum creatinine $(\mathrm{mg} / \mathrm{dl})$ & P value \\
\hline C & 7 & $4.73 \pm 0.32$ & $<0.001$ \\
E & 7 & $1.82 \pm 0.03$ & \\
\hline
\end{tabular}

Data were expressed as Mean \pm SE. Statistical analysis were done by One-way ANOVA test and Bonferroni test was performed to compare between groups. Experiment-II Group C (Experimental Control) Group D (Low dose kalajira) Group $\mathrm{E}$ (High dose kalajira); $\mathrm{n}=$ Total number of subjects $=$ Significant at $\mathrm{P}<0.05$; Not significant $(\mathrm{P}>0.05)$ 
Table-III

Comparison of serum creatinine between Group D and Group E.

\begin{tabular}{lccc}
\hline Group & Number of rats & Mean serum & \\
\hline $\mathrm{D}$ & 7 & $3.59 \pm 0.15$ & P value \\
$\mathrm{E}$ & 7 & $1.82 \pm 0.03$ & 0.002 \\
\hline
\end{tabular}

Data were expressed as Mean $\pm \mathrm{SE}$. Statistical analysis were done by One-way ANOVA test and Bonferroni test was performed to compare between groups. Experiment-II, Group D (Low dose kalajira) Group E(High dose kalajira); $\mathrm{n}=$ Total number of subjects = Significant at $\mathrm{P}<0.05$; Not significant $(\mathrm{P}>0.05)$

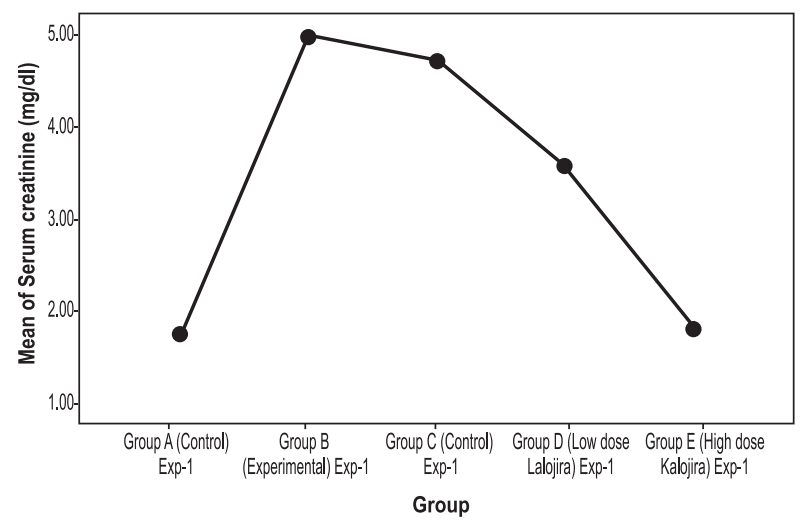

Fig. 1 : Line diagram showing the mean serum creatinine $(\mathrm{mg} / \mathrm{dl})$ in different study group

\section{Discussion}

The present study was aimed to ameliorate the renal toxicity of a commonly used aminoglycoside antibiotic (gentamicin) with the administration of Nigella sativa Linn. (Kalajira), widely used herbal spice of Southeast Asia and the Middle East, which has been renowned for its various beneficial effects in the human. Gentamicin was administered subcutaneously on the nape of the neck of Adult male rats (weighing 200-230g) at a dose of 100 $\mathrm{mg} / \mathrm{kg}$ body weight/day for 9 days(sacrificed on day -10). Nephrotoxicity and the ameliorating effects were estimated by biochemical findings.

Experiment I was conducted prior to the commencement of the main study (Experiment II). In Experiment I, Group A served as control group and they will be provided with normal rat diet and water ad libitum for 9 days and were sacrificed on $10^{\text {th }}$ day. Group B is gentamicin treated group. Rats of this group were given gentamicin subcutaneously $(100 \mathrm{mg} / \mathrm{kg}$ body weight/day) for 9 days. The rats were also allowed usual rat diet and water ad libitum for the same days (first week) and were sacrificed on day 10. In Experiment II , n-hexane extract of N. sativa Linn. (kalajira) was administered orally through Ryle's tube at a dose of $10 \mathrm{mg} / \mathrm{kg}$ body weight/day to the group D and $20 \mathrm{mg} / \mathrm{kg}$ body weight/day to the group $\mathrm{E}$ of adult male rats for 9 days. All the rats were adult, male and of same species with the expectation that the interpretation of the findings could be easier and moreover, intergroup comparisons of the findings would be more relevant. In the present study, male rats were selected because male rats are believed to be more prone to gentamicin-induced nephrotoxicity than female rats (Bennett et al.,1982).

The control group (group A) of rats were given normal rat diet and water ad libitum and sacrificed on day 10. The effects on serum creatinine concentrations were observed. The values obtained from the above parameters were similar to values in normal adult rats reported in earlier studies (Abul-Nasr et al., 2001;).

Group B (gentamicin treated, sacrificed on day10) was compared with group A (control), it was found that there was significant increase $(\mathrm{P}<0.001)$ of serum creatinine concentrations. These findings also indicate that gentamicin administration has induced damage to the renal tubules which has been the reason of increased creatinine level obtained in our samples. The above mentioned authors have reported similar biochemical changes and have described them as suggestive of nephrotoxicity.

In group $\mathrm{C}$ (gentamicin-treated, sacrificed on day 10) taken as control group of experiment II could be obtained in biochemical variables . 
The effects of low and high dose administration of N.sativa Linn.(Kalajira) on gentamicininduced nephrotoxic rats were obtained by estimation of serum creatinine in group D (gentmicin and low dose N. sativa concomitantly) and group $\mathrm{E}$ (gentamicin and high dose $\mathrm{N}$. sativa concomitantly). In our observation, group $\mathrm{E}$ had showed significant $(\mathrm{P}<0.001)$ decrease in serum creatinine compared to those observed in group D.

Cumulating these evidences together, the findings of the pilot study suggest that N. sativa Linn.(kalajira) was able to produce considerable alleviation from the nephrotoxic action of gentamicin in adult male rats.

In Experiment II, effect of gentamicin and the effects of the $n$-hexane extract of N.sativa on renal biochemical parameters of rats were observed, i.e. groups C, D and E.

There was also significant increase of serum creatinine concentrations when compared with vehicle control (group A). These findings were similar to those of previous workers (Hayashi et al., 1988 ;Pedraza-Chaverri et al ., 2000;). Serum creatinine is more sensitive indicator renal dysfunction and the elevation suggests renal proximal tubule damage (Davidson et al., 1999).

\section{Conclusion:}

The results of this study indicate that oral administration of $\mathrm{n}$-hexane extract of $\mathrm{N}$. sativa was able to produce considerable alleviation from the nephrotoxic action of gentamicin in rats. The best amelioration was obtained by high dose treatment. Low dose treatment brought out the least amelioration of them all. Future works could be better directed towards obtaining the specific ingredient and the specific mechanism(if any, other than the antioxidant action). We are hopeful that complete amelioration might not be impossible.

\section{References:}

1. Abbas M T,Zayni M.Effect of Garlic Oil on Gentamicin Induced Hepatorenal Toxicity in rats. Journal of KerbalaUniversity , Scientific . 2013;11(2):109-117.

2. Abul-Ezz SR, Walker PD, Shah SV. Role of glutathione in an animal model of myoglobinuric acute renal failure. Proc. Natl Acad Si 1991; 88;9833-37.

3. Ahmad A, Asif Husain.A review on therapeutic potential of Nigella sativa: A miracle herb. Asian Pac J Trop Biomed 2013; 3(5): 337-352.

4. Alam SD, Reddy KS. Evaluation of anti-diabetic and anti-lipidimic potential of kalongi sugar powder water extract in stz induced diabetic rats. International Journal of Pharmacy and Pharmaceutical Sciences 2013;5:94-96.

5. Bennett PN, Brown MJ. Antibacterial drugs. IN: Clinical pharmacology. $9^{\text {th }}$ ed. London : Churchill Living stone 2003: pp. 2015 - 35.

6. Chambers HF. The aminoglycosides. In : Hardman JG, Limbird LE, editors. Goodman and gilman's the pharmacological basis of therapeutics. $10^{\text {th }}$ ed. New York : Mc Graw-Hill Book Company, Inc, 2001 ; pp. 1219-31.

7. Chambers HF. Aminoglycosides and spectinomycin. In : Katzung : Basic and clinical pharmacology. $9^{\text {th }}$ ed. New York : Lange and Mc Grow-Hill 2004 : pp. 784-91. 